\title{
Development of cerebral vasospasm following traumatic intracranial hemorrhage: incidence, risk factors, and clinical outcomes
}

\author{
Alis J. Dicpinigaitis, BA, ${ }^{1}$ Eric Feldstein, MD, ${ }^{2}$ Nitesh Damodara, MD, ${ }^{2}$ Jared B. Cooper, MD, ${ }^{2}$ \\ Steven D. Shapiro, MD, ${ }^{2}$ Haris Kamal, MD, ${ }^{2}$ Merritt D. Kinon, MD, ${ }^{2}$ Jared Pisapia, MD, MTR, ${ }^{2}$ \\ Jon Rosenberg, MD, ${ }^{3}$ Chirag D. Gandhi, MD, ${ }^{2}$ and Fawaz AI-Mufti, MD²
}

${ }^{1}$ School of Medicine, New York Medical College; ' ${ }^{2}$ epartment of Neurosurgery, Westchester Medical Center; and ${ }^{3}$ Department of Neurology, Westchester Medical Center, Valhalla, New York

\begin{abstract}
OBJECTIVE Limited evidence exists characterizing the incidence, risk factors, and clinical associations of cerebral vasospasm following traumatic intracranial hemorrhage $(\mathrm{tICH})$ on a large scale. Therefore, the authors sought to use data from a national inpatient registry to investigate these aspects of posttraumatic vasospasm (PTV) to further elucidate potential causes of neurological morbidity and mortality subsequent to the initial insult.
\end{abstract}

METHODS Weighted discharge data from the National (Nationwide) Inpatient Sample from 2015 to 2018 were queried to identify patients with $\mathrm{tICH}$ who underwent diagnostic angiography in the same admission and, subsequently, those who developed angiographically confirmed cerebral vasospasm. Multivariable logistic regression analysis was performed to identify significant associations between clinical covariates and the development of vasospasm, and a tICH vasospasm predictive model (tICH-VPM) was generated based on the effect sizes of these parameters.

RESULTS Among 5880 identified patients with $\mathrm{tlCH}, 375$ developed PTV corresponding to an incidence of $6.4 \%$. Multivariable adjusted modeling determined that the following clinical covariates were independently associated with the development of PTV, among others: age (adjusted odds ratio [aOR] 0.98, 95\% Cl 0.97-0.99; $p<0.001$ ), admission Glasgow Coma Scale score < 9 (aOR 1.80, 95\% Cl 1.12-2.90; $p=0.015)$, intraventricular hemorrhage (aOR 6.27, 95\% $\mathrm{Cl} 3.49-11.26 ; p<0.001$ ), tobacco smoking (aOR 1.36, 95\% Cl 1.02-1.80; $p=0.035$ ), cocaine use (aOR 3.62, 95\% Cl 1.97-6.63; $p<0.001$ ), fever (aOR 2.09, 95\% Cl 1.34-3.27; $p=0.001$ ), and hypokalemia (aOR 1.62, 95\% Cl 1.26-2.08; $p$ $<0.001$ ). The tICH-VPM achieved moderately high discrimination, with an area under the curve of 0.75 (sensitivity $=0.61$ and specificity $=0.81$ ). Development of vasospasm was independently associated with a lower likelihood of routine discharge (aOR 0.60, 95\% Cl 0.45-0.78; $p<0.001)$ and an extended hospital length of stay (aOR 3.53, 95\% $\mathrm{Cl} 2.78-4.48$; $p<0.001)$, but not with mortality.

CONCLUSIONS This population-based analysis of vasospasm in tICH has identified common clinical risk factors for its development, and has established an independent association between the development of vasospasm and poorer neurological outcomes.

https://thejns.org/doi/abs/10.3171/2021.12.FOCUS21668

KEYWORDS vasospasm; intracranial hemorrhage; trauma; subarachnoid hemorrhage; database; predictive model; decision-tree analysis

$\mathrm{A}$ LTHOUGH cerebral vasospasm represents an area of intensive investigation in the setting of aneurysmal subarachnoid hemorrhage (SAH), comparatively less is known of this entity following traumatic intracranial hemorrhage (tICH). Documented rates of incidence range widely $(5 \%-63 \%)$ as a result of minimal surveillance for its development and the application of varied imaging and diagnostic modalities for its detection. ${ }^{1}$ The etiology of posthemorrhagic vasoconstriction of the cerebral vasculature remains unclear, while the prognostic

ABBREVIATIONS aOR = adjusted odds ratio; $\mathrm{DCI}=$ delayed cerebral ischemia; GCS = Glasgow Coma Scale; HCUP = Healthcare Cost and Utilization Project; IVH = intraventricular hemorrhage; LOS = length of stay; NIS = National (Nationwide) Inpatient Sample; PTV = posttraumatic vasospasm; ROC = receiver operating characteristic; $\mathrm{SAH}=$ subarachnoid hemorrhage; $\mathrm{TBI}=$ traumatic brain injury; $\mathrm{tICH}=$ traumatic intracranial hemorrhage; VPM = vasospasm predictive model.

SUBMITTED November 1, 2021. ACCEPTED December 22, 2021.

INCLUDE WHEN CITING DOI: 10.3171/2021.12.FOCUS21668. 
significance of vasospasm in traumatic brain injury (TBI) has largely been unexamined. As the incidence of TBI continues to increase in the US, ${ }^{2}$ it is paramount to further elucidate potential causes of neurological morbidity and mortality subsequent to the initial insult. This study uses data from a national inpatient registry to characterize the incidence, risk factors, and clinical outcomes of posttraumatic vasospasm (PTV) on a large scale.

\section{Methods \\ Data Source}

The National (Nationwide) Inpatient Sample (NIS), developed and maintained by the Healthcare Cost and Utilization Project (HCUP), is among the largest publicly accessible inpatient care databases in the US. Yearly unweighted data approximate 7,000,000 patients, reflecting a $20 \%$ stratified sample of all HCUP-participating community hospitals nationally. The large sample size afforded by the NIS allows for substantive inquiry into healthcare utilization, access, charges, quality, and outcomes, as well as reliable reproduction of national estimates annually. Data elements include demographic characteristics, hospital and regional information, diagnoses, procedures, and discharge disposition for all documented patients. More information regarding the NIS and data access can be found at https://www.hcup-us.ahrq.gov. Given the public accessibility and de-identified nature of the information in this database, this study did not meet the requirements for institutional review board approval. For the same reason, patient consent was not required.

\section{Patient Selection and Cohort Development}

International Classification of Diseases, Tenth Revision, Clinical Modification (ICD-10-CM) codes were used to identify patients with admission diagnoses for intracranial injury (ICD-10-CM S06.X) from 2015 (fourth quarter, October through December) to 2018 and included focal traumatic hemorrhage of the cerebrum, brainstem, and cerebellum (ICD-10-CM S06.3X), traumatic SAH (S06.6X), and intraventricular hemorrhage (IVH) due to trauma (ICD-10-CM I61.5 with S06.X). Patients were included if they underwent diagnostic cerebral angiography (ICD-10-PCS B31XXXX) during the index hospitalization for their trauma admission; this was to ensure that radiographic evidence was used to confirm the presence or absence of cerebral vasospasm (ICD-10-CM I67.84), which was identified in accordance with previous literature. ${ }^{3,4}$ Intracranial arterial angioplasty for the treatment of vasospasm was identified using the relevant procedural identifier (ICD-10-PCS 037GXXX). The finalized cohort was queried to identify demographic characteristics (age and female sex) as well as comorbid conditions and clinical variables associated with the development of vasospasm.

\section{Clinical Endpoints}

The primary clinical endpoint of this analysis was the development of PTV. Clinical outcomes (routine discharge to home or self-care, mortality, and hospital length of stay [LOS]) were evaluated as a secondary endpoint.

\section{Statistical Analysis}

All analyses were performed within a complex samples function with appropriate stratum and cluster variables and discharge weights per HCUP guidelines to account for NIS sampling design and, ultimately, to yield accurate national estimates. Descriptive statistics were performed to measure variation in baseline demographic and clinical characteristics as well as in outcomes between patients with traumatic hemorrhage who developed PTV and those who did not develop PTV. Dichotomous variables were assessed using Pearson's chi-square test and are presented as count (\%), while estimated marginal means for continuous baseline parameters were computed using the t-test, within a complex samples general linear model, and reported with SEM. Statistical significance was evaluated at $\mathrm{p}<$ 0.05 for univariable comparisons.

A multivariable logistic regression model composed of all baseline clinical parameters identified significant independent associations with the development of PTV. Beta $(\beta)$ coefficients representing effect sizes of significant parameters $(\mathrm{x})$ were generated to construct a tICH vasospasm predictive model (tICH-VPM) described by the following expression:

$$
\sum_{i=0}^{n} \beta_{0} x_{0}+\beta_{1} x_{1} \ldots \beta_{n} x_{n}
$$

Receiver operating characteristic (ROC) curve analysis was subsequently performed to evaluate the discriminatory capacity of this model, while the Youden index was used to identify sensitivity and specificity based on the coordinate points of the highest combination of these two parameters on the ROC curve. Finally, decision-tree analysis was performed using a chi-square automatic interaction detection (CHAID) algorithm using all baseline parameters as candidate variables for node construction with adjusted Bonferroni testing for decision-making.

\section{Results}

Among 5880 identified patients with tICH, 375 developed PTV for an overall incidence of 6.4\%; $75(20 \%)$ of these patients were subsequently treated with cerebral angioplasty. Univariable comparison of baseline characteristics and clinical variables between patients who developed PTV and those who did not develop PTV is shown in Table 1. Following multivariable logistic regression analysis, the following clinical covariates were independently associated with the development of PTV: age (adjusted odds ratio [aOR] 0.98, 95\% CI 0.97-0.99; $\mathrm{p}<0.001$ ), female sex (aOR 1.80, 95\% CI 1.43-2.27; $\mathrm{p}<0.001$ ), admission Glasgow Coma Scale (GCS) score < 9 (aOR 1.80, 95\% CI $1.12-2.90 ; \mathrm{p}=0.015)$, SAH (aOR $2.46,95 \%$ CI $1.45-$ $4.12 ; \mathrm{p}=0.001$ ), IVH (aOR 6.27, 95\% CI 3.49-11.26; $\mathrm{p}<$ 0.001 ), obesity (aOR $2.34,95 \%$ CI 1.65-3.37; p < 0.001), diabetes mellitus (aOR 0.45, 95\% CI 0.31-0.67; $\mathrm{p}<0.001$ ), hypertension (aOR 1.77, 95\% CI 1.36-2.30; $\mathrm{p}<0.001$ ), 
TABLE 1. Comparison of baseline demographic and clinical characteristics, hospital course, and outcomes between patients with $\mathrm{tICH}$ with and without development of cerebral vasospasm

\begin{tabular}{|c|c|c|c|c|}
\hline & $\begin{array}{l}\text { Total Cohort } \\
(n=5880)\end{array}$ & $\begin{array}{c}\text { Vasospasm } \\
(\mathrm{n}=375,6.4 \%)\end{array}$ & $\begin{array}{c}\text { No Vasospasm } \\
(n=5505,93.6 \%)\end{array}$ & $p$ Value \\
\hline \multicolumn{5}{|l|}{$\begin{array}{l}\text { Baseline demographic \& clinical } \\
\text { characteristics }\end{array}$} \\
\hline Mean age, yrs (SE) & $55.9(0.3)$ & $51.6(0.9)$ & $56.2(0.3)$ & $<0.001$ \\
\hline Female sex & $2285(38.9)$ & $190(50.7)$ & 2095 (38.1) & $<0.001$ \\
\hline Admission GCS score $<9$ & $200(3.4)$ & $25(6.7)$ & $175(3.2)$ & $<0.001$ \\
\hline Intraparenchymal hemorrhage & $1100(18.7)$ & $50(13.3)$ & 1050 (19.1) & 0.006 \\
\hline $\mathrm{SAH}$ & $4995(84.9)$ & $320(85.3)$ & $4675(84.9)$ & 0.830 \\
\hline IVH & $230(3.9)$ & $45(12.0)$ & $185(3.4)$ & $<0.001$ \\
\hline \multicolumn{5}{|l|}{ Comorbidities } \\
\hline Obesity & $390(6.6)$ & $50(13.3)$ & $340(6.2)$ & $<0.001$ \\
\hline Diabetes mellitus & $1060(18.0)$ & $40(10.7)$ & $1020(18.5)$ & $<0.001$ \\
\hline Hypertension & $3230(54.9)$ & $225(60.0)$ & $3005(54.6)$ & 0.041 \\
\hline Congestive heart failure & $575(9.8)$ & $40(10.7)$ & $535(9.7)$ & 0.550 \\
\hline Chronic renal disease & 485 (8.2) & $25(6.7)$ & $460(8.4)$ & 0.250 \\
\hline Hyperlipidemia & $1580(26.9)$ & $85(22.7)$ & $1495(27.2)$ & 0.058 \\
\hline \multicolumn{5}{|l|}{ Substance use } \\
\hline Tobacco smoking & $900(15.3)$ & $80(21.3)$ & $820(14.9)$ & 0.001 \\
\hline Cocaine & $95(1.6)$ & $15(4.0)$ & $80(1.5)$ & $<0.001$ \\
\hline Alcohol & $1135(19.3)$ & $70(18.7)$ & 1065 (19.3) & 0.747 \\
\hline \multicolumn{5}{|l|}{ Laboratory \& vital parameters } \\
\hline Leukocytosis & $475(8.1)$ & $45(12.0)$ & $430(7.8)$ & 0.004 \\
\hline Acute anemia & NR & NR & NR & $<0.001$ \\
\hline Hyperglycemia & $275(4.7)$ & NR & $265(4.8)$ & 0.057 \\
\hline Hyponatremia & $1040(17.7)$ & $85(22.7)$ & $955(17.3)$ & 0.009 \\
\hline Hypernatremia & 710 (12.1) & $60(16.0)$ & $650(11.8)$ & 0.016 \\
\hline Hypokalemia & $1115(19.0)$ & $120(32.0)$ & $995(18.1)$ & $<0.001$ \\
\hline Fever & $215(3.7)$ & $30(8.0)$ & $185(3.4)$ & $<0.001$ \\
\hline Acute elevated blood pressure & $220(3.7)$ & $40(10.7)$ & $180(3.3)$ & $<0.001$ \\
\hline \multicolumn{5}{|l|}{ Clinical outcomes } \\
\hline Routine discharge & $2075(35.3)$ & $85(22.7)$ & 1990 (36.1) & $<0.001$ \\
\hline In-hospital mortality & $630(10.7)$ & $50(13.3)$ & $580(10.5)$ & 0.091 \\
\hline Mean LOS, days (SE) & $13.2(0.2)$ & $18.8(0.5)$ & $12.8(0.2)$ & $<0.001$ \\
\hline
\end{tabular}

$\mathrm{NR}=$ not reported due to low case number $(\mathrm{n}<11)$.

Values represent the number of patients (\%) and were compared using chi-square analysis. Continuous parameters are presented as mean (SE) and were compared by t-test. Boldface type indicates statistical significance evaluated at $p<$ 0.05 for univariable comparisons.

congestive heart failure (aOR 1.67, 95\% CI 1.13-2.45; p $=0.009)$, tobacco smoking (aOR 1.36, 95\% CI 1.02-1.80; $\mathrm{p}=0.035)$, cocaine use (aOR 3.62, 95\% CI 1.97-6.63; p $<0.001$ ), fever (aOR 2.09, 95\% CI 1.34-3.27; $\mathrm{p}=0.001$ ), hyperglycemia (aOR 0.31, 95\% CI 0.16-0.60; $\mathrm{p}=0.001$ ), hypernatremia (aOR 1.42, 95\% CI 1.03-1.94; $\mathrm{p}=0.032$ ), hypokalemia (aOR 1.62, 95\% CI 1.26-2.08; p < 0.001), acute anemia (aOR 11.47, 95\% CI 3.04-43.28; p < 0.001), and acute elevated blood pressure (aOR 3.23, 95\% CI 2.15-4.87; p < 0.001) (Table 2).

The tICH-VPM achieved an area under the ROC curve of 0.748 (sensitivity $=0.612$, specificity $=0.810$, and Youden index $=0.422$ ) (Fig. 1). In decision-tree analysis,
IVH served as the parent node while age subgroups and hypokalemia were secondary nodes in the clinical algorithm of risk factors for development of PTV (Fig. 2).

Patients who developed PTV were routinely discharged at a rate of $22.7 \%$, had a mean LOS of 18.8 days, and died during hospitalization at a rate of $13.3 \%$ (Table 1). Following multivariable logistic regression analysis, PTV was independently associated with lower likelihood of a routine discharge (aOR 0.60, 95\% CI 0.45-0.78; p < 0.001) and an extended hospital LOS (evaluated at the 75 th percentile for durations of hospitalization in the aggregate cohort, corresponding to 18 days) (aOR 3.53, 95\% CI 2.78-4.48; $\mathrm{p}$ $<0.001$ ), but not with mortality (Table 3 ). 
TABLE 2. Multivariable analysis to determine associations of clinical characteristics with development of cerebral vasospasm

\begin{tabular}{lccr}
\hline \multicolumn{1}{c}{ Clinical Characteristic } & $\beta$ Coefficient & aOR $(95 \%$ Cl) & $p$ Value \\
\hline Age & -0.02 & $0.98(0.97-0.99)$ & $<0.001$ \\
\hline Female sex & 0.59 & $1.80(1.43-2.27)$ & $<0.001$ \\
\hline Admission GCS score $<9$ & 0.59 & $1.80(1.12-2.90)$ & 0.015 \\
\hline Intraparenchymal & - & $1.11(0.76-1.62)$ & 0.604 \\
hemorrhage & & & \\
\hline SAH & 0.90 & $2.46(1.45-4.12)$ & 0.001 \\
\hline IVH & 1.84 & $6.27(3.49-11.26)$ & $<0.001$ \\
\hline Obesity & 0.86 & $2.34(1.65-3.37)$ & $<0.001$ \\
\hline Diabetes mellitus & -0.79 & $0.45(0.31-0.67)$ & $<0.001$ \\
\hline Hypertension & 0.57 & $1.77(1.36-2.30)$ & $<0.001$ \\
\hline Congestive heart failure & 0.51 & $1.67(1.13-2.45)$ & 0.009 \\
\hline Chronic renal disease & - & $1.04(0.65-1.67)$ & 0.863 \\
\hline Hyperlipidemia & - & $0.83(0.62-1.12)$ & 0.227 \\
\hline Tobacco smoking & 0.31 & $1.36(1.02-1.80)$ & 0.035 \\
\hline Cocaine use & 1.29 & $3.62(1.97-6.63)$ & $<0.001$ \\
\hline Alcohol use & - & $0.75(0.55-1.00)$ & 0.053 \\
\hline Leukocytosis & - & $1.19(0.82-1.71)$ & 0.361 \\
\hline Fever & 0.74 & $2.09(1.34-3.27)$ & 0.001 \\
\hline Hyperglycemia & -1.17 & $0.31(0.16-0.60)$ & 0.001 \\
\hline Hyponatremia & - & $1.30(0.99-1.71)$ & 0.056 \\
\hline Hypernatremia & 0.35 & $1.42(1.03-1.94)$ & $\mathbf{0 . 0 3 2}$ \\
\hline Hypokalemia & 0.48 & $1.62(1.26-2.08)$ & $<0.001$ \\
\hline Acute anemia & 2.44 & $11.47(3.04-43.28)$ & $<0.001$ \\
\hline Acute elevated blood & 1.17 & $3.23(2.15-4.87)$ & $<0.001$ \\
pressure & & & \\
\hline Efectszesof & & & \\
\hline
\end{tabular}

Effect sizes of baseline clinical covariates from unadjusted analysis for associations with development of vasospasm presented as aORs with $95 \% \mathrm{Cls}$ following multivariable logistic regression analysis with all baseline clinical characteristics as model covariates. Boldface type indicates statistical significance evaluated at $p<0.05$. $\beta$ coefficients from significant parameters were subsequently used to construct the IICH-VPM.

\section{Discussion}

The incidence of PTV has historically been difficult to ascertain due to lack of routine assessment. Since it is often not assessed unless there are clinical indications, including delayed cerebral ischemia (DCI), asymptomatic vasospasm is rarely detected. Furthermore, SAH, a major predictor for the development of cerebral vasospasm, is present in as many as $60 \%$ of patients with TBI. ${ }^{1,5}$ Previously, it has been shown that vasospasm occurs in as little as $5 \%$ and as much as $18.6 \%$ of TBI-associated ICH. Now, with the improvement of widespread imaging modalities, the incidence has been estimated to be as high as 63\%, although with a larger risk of false positives. In this study, we found the incidence of PTV to be $6.4 \%$, consistent with rates reported from traditional diagnostic imaging modalities. $^{6}$

PTV has been thought to develop similarly to the better-understood pathophysiology of post-SAH vasospasm, whereby the extravasation of blood into the subarachnoid

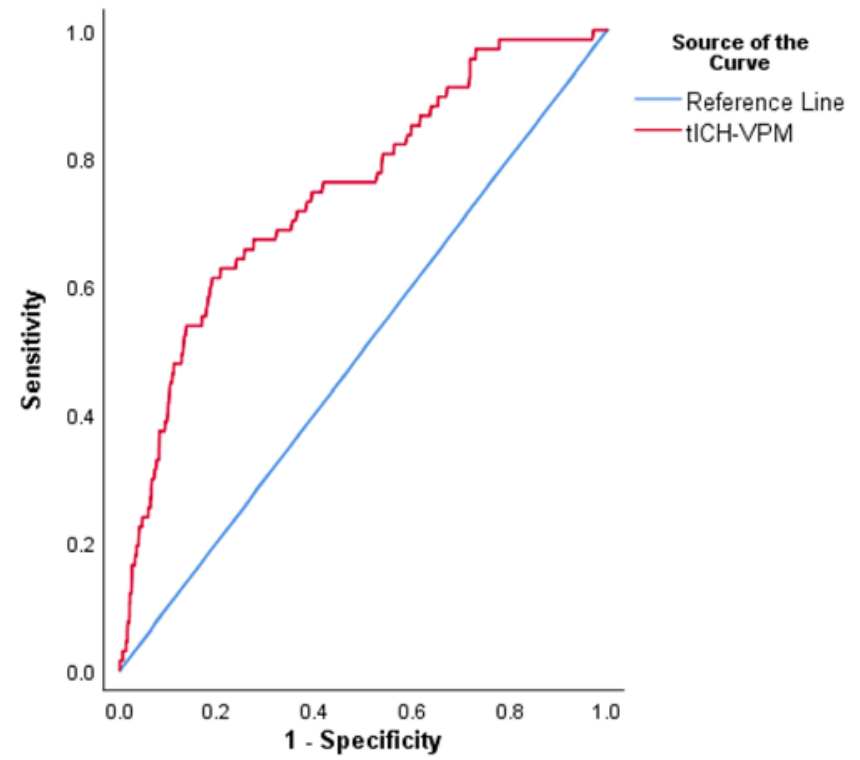

FIG. 1. ROC curve analysis for the tICH-VPM.

space and resultant release of neuroinflammatory substances through its breakdown cause direct irritation to the cerebral vasculature. ${ }^{7}$ However, this does not explain the incidence of vasospasm in patients lacking SAH after trauma. ${ }^{8}$ In this case, in vitro studies have found that direct trauma through pulling and shearing forces can induce vasospasm as well. ${ }^{9,10}$ Symptomatic vasospasm can be readily caught by consistent neurological examinations, although at that point the effects are often irreversible. ${ }^{11}$

Traditional comorbid risk factors found in small cohort and population-based studies include high-grade $\mathrm{SAH}$, intracerebral hematoma, and epidural and subdural hematoma formation. ${ }^{1}$ Clinical presentations associated with the development of PTV include fever, elevated Injury Severity Scores, decreasing GCS, and the extent of involved cerebrum following TBI. ${ }^{12-14}$ Based on multivariable analysis, we found female sex to be a demographic predictor of PTV, while decreasing age was associated with increased odds of developing PTV. Clinically, the presence of IVH, $\mathrm{SAH}$, and an admission GCS score $<9$ were found to be predictive of PTV, in descending order. In terms of comorbidities, anemia, cocaine use, acute elevated blood pressure, obesity, fever, hypokalemia, hypertension, congestive heart failure, hypernatremia, and tobacco use were associated with increased risk of developing PTV, in descending order as well.

In a decision-tree algorithm, IVH was found to be the parent node in establishing the risk of developing PTV. Case reports on the incidence of vasospasm in patients with ruptured arteriovenous malformations have found IVH to be linked with delayed vasospasm as well as DCI 14 days after the initial hemorrhage, perhaps due to stasis of lysed blood products in the CSF space. ${ }^{15-17}$ In a previous analysis of one institution's database on ICH, Amuluru et al. found the incidence of vasospasm in instances of IVH to be $13.9 \%$, with a subsequent DCI incidence of $11.1 \%$, constituting an independent pathophysiological risk fac- 


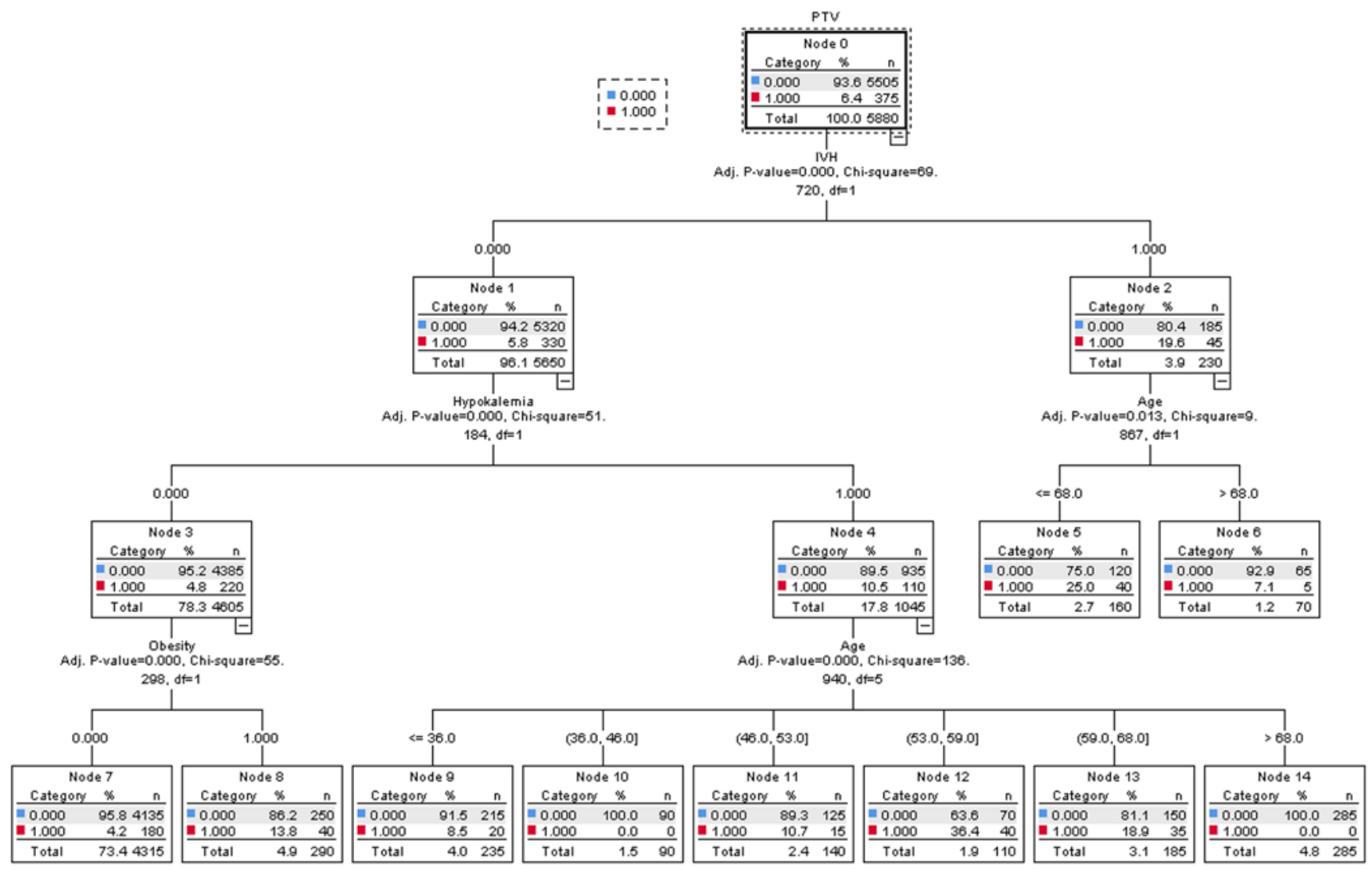

FIG. 2. Decision-tree algorithm for the development of cerebral vasospasm following $\mathrm{tICH}$.

tor. ${ }^{18}$ Interestingly, IVH was a far more robust independent predictor of vasospasm in comparison with $\mathrm{SAH}$, the hemorrhage type traditionally associated with its development.

Potential biomarkers for the development of PTV identified in this study include fever, hypokalemia, and hypernatremia, all of which were independent predictors in multivariable modeling. In a previous analysis of biomarkers for the development of vasospasm after SAH, a study that used the Nationwide Readmissions Database found hypernatremia, hypotension, and anemia to be correlated with vasospasm development. The authors acknowledged that poor hemodynamics leading to cerebral hypoxia can result in DCI and linked fever and leukocytosis with the degree of clot burden and vasospasm in these patients. ${ }^{3}$ Hypokalemia does not have a clear clinical relationship with the development of vasospasm, although previous analyses have postulated electrolyte-induced imbalances in sympathetic activation and other autonomic processes as a potential explanation. Conversely, perhaps sympathetic activation secondary to brain injury, and a known pathogenesis of vasospasm in patients with $\mathrm{SAH}$, also contributes to the development of hypokalemia in these patients. ${ }^{3,19}$

While we identified many similar comorbidities of TBI that are associated with vasospasm as have similarly been shown, including obesity, hypertension, and tobacco smoking, we identified hyperglycemia and diabetes mellitus as protective factors. Although it has been shown that glycemic indices can predict outcomes after $\mathrm{SAH}$, with a higher incidence of complications and mortality, the link with the establishment of vasospasm is less clear. ${ }^{20}$ Perhaps the long-term microvascular effects of poor glycemic control and development of cholesterol plaques and vessel narrowing provide a scaffolding which counters the vasospastic properties of blood breakdown products; however, this must be further investigated.

DCI, induced by the development of vasospasm, is one of the most significant causes of morbidity and mortality following an initial SAH insult. In one cohort study out of Columbia University, DCI was second only to rebleeding as the leading cause of death secondary to the initial bleed in SAH. ${ }^{21}$ The link between vasospasm and clinical

TABLE 3. Multivariable analysis to determine the association of cerebral vasospasm with clinical outcomes

\begin{tabular}{lcr}
\hline \multicolumn{1}{c}{ Clinical Outcome } & aOR $(95 \% \mathrm{Cl})$ & p Value \\
\hline Routine discharge & $0.60(0.45-0.78)$ & $<0.001$ \\
\hline In-hospital mortality & $1.31(0.92-1.86)$ & 0.130 \\
\hline Extended LOS (75th percentile) & $3.53(2.78-4.48)$ & $<0.001$ \\
\hline
\end{tabular}

Effect sizes of cerebral vasospasm for clinical outcomes presented as aORs with $95 \% \mathrm{Cls}$ following multivariable logistic regression analysis with all baseline clinical characteristics as model covariates. Boldface type indicates statistical significance following Bonferroni correction for multiple comparisons. 
outcomes remains poorly understood in the setting of TBI. It has been documented that, while early PTV is linked with poorer outcomes, late PTV and overall PTV have no association. ${ }^{22}$ This can be due, in part, to the association of early PTV with the development of DCI and late PTV being an asymptomatic finding. ${ }^{23}$ Our results show that while the presence of vasospasm leads to less routine discharge and an extended hospital LOS in the TBI cohort, it has no overall increase in the rate of in-hospital mortality. Nonetheless, vasospasm emerged as an independent predictor of poorer neurological outcomes irrespective of the severity of TBI and comorbidity burden.

\section{Limitations}

The primary limitations of this investigation are those traditionally associated with retrospective studies that use administrative registry data, by which all clinical variables are derived from billing codes assigned following patient discharge. Cerebral vasospasm was identified using a singular billing code, and, thus, severity and specific localization could not be evaluated in this analysis. Moreover, diagnosis codes do not carry a temporal qualifier, which prevented documentation of when vasospasm occurred and, therefore, introduces potential inaccuracy with clinical covariates included in this analysis (such as laboratory markers) due to the inability to ascertain whether they occurred before or after the development of vasospasm. These limitations notwithstanding, the large cohort size and utilization of national data underscore the generalizability of the findings of our study, which, to our knowledge, is the first of its kind to evaluate vasospasm in $\mathrm{tICH}$ on a large scale.

\section{Conclusions}

This large-scale analysis using national registry data identified risk factors for the development of PTV in $\mathrm{tICH}$ and employed predictive modeling and decision-tree algorithms to aid in the identification of patients at risk. Moreover, it has provided evidence for worse neurological outcomes in patients developing PTV. Further investigation is warranted for management strategies and optimal modalities for detection of PTV in patients with tICH.

\section{References}

1. Al-Mufti F, Amuluru K, Changa A, et al. Traumatic brain injury and intracranial hemorrhage-induced cerebral vasospasm: a systematic review. Neurosurg Focus. 2017;43(5): E14.

2. GBD 2016 Traumatic Brain Injury and Spinal Cord Injury Collaborators. Global, regional, and national burden of traumatic brain injury and spinal cord injury, 1990-2016: a systematic analysis for the Global Burden of Disease Study 2016. Lancet Neurol. 2019;18(1):56-87.

3. Rumalla K, Lin M, Ding L, et al. Risk factors for cerebral vasospasm in aneurysmal subarachnoid hemorrhage: a population-based study of 8346 patients. World Neurosurg. 2021;145:e233-e241.

4. Kaculini C, Wallace DJ, Haywood AE, et al. Protective effects of obstructive sleep apnea on outcomes after subarachnoid hemorrhage: a nationwide analysis. Neurosurgery. 2020; 87(5):1008-1015.

5. Armin SS, Colohan ART, Zhang JH. Vasospasm in traumatic brain injury. Acta Neurochir Suppl (Wien). 2008;104(13):421425.

6. Vora YY, Suarez-Almazor M, Steinke DE, Martin ML, Findlay JM. Role of transcranial Doppler monitoring in the diagnosis of cerebral vasospasm after subarachnoid hemorrhage. Neurosurgery. 1999;44(6):1237-1248.

7. Wilkins RH, Odom GL. Intracranial arterial spasm associated with craniocerebral trauma. J Neurosurg. 1970;32(6): 626-633.

8. Oertel M, Boscardin WJ, Obrist WD, et al. Posttraumatic vasospasm: the epidemiology, severity, and time course of an underestimated phenomenon: a prospective study performed in 299 patients. J Neurosurg. 2005;103(5):812-824.

9. Kramer DR, Winer JL, Pease BAM, Amar AP, Mack WJ. Cerebral vasospasm in traumatic brain injury. Neurol Res Int. 2013;2013:415813.

10. Arutiunov AI, Baron MA, Majorova NA. The role of mechanical factors in the pathogenesis of short-term and prolonged spasm of the cerebral arteries. J Neurosurg. 1974; 40(4):459-472.

11. Westermaier T, Pham M, Stetter C, et al. Value of transcranial Doppler, perfusion-CT and neurological evaluation to forecast secondary ischemia after aneurysmal SAH. Neurocrit Care. 2014;20(3):406-412.

12. Shahlaie K, Keachie K, Hutchins IM, et al. Risk factors for posttraumatic vasospasm. J Neurosurg. 2011;115(3):602-611.

13. O'Brien NF, Maa T, Yeates KO. The epidemiology of vasospasm in children with moderate-to-severe traumatic brain injury. Crit Care Med. 2015;43(3):674-685.

14. Zubkov AY, Lewis AI, Raila FA, Zhang J, Parent AD. Risk factors for the development of post-traumatic cerebral vasospasm. Surg Neurol. 2000;53(2):126-130.

15. Tseng WL, Tsai YH. Vasospasm after intraventricular hemorrhage caused by arteriovenous malformation. Asian $J$ Neurosurg. 2015;10(2):114-116.

16. Maeda K, Kurita H, Nakamura T, et al. Occurrence of severe vasospasm following intraventricular hemorrhage from an arteriovenous malformation. Report of two cases. J Neurosurg. 1997;87(3):436-439.

17. Park BS, Won YS, Choi CS, Kim BM. Severe symptomatic vasospasm following intraventricular hemorrhage from arteriovenous fistula. J Korean Neurosurg Soc. 2009;45(5): 300-302.

18. Amuluru K, Al-Mufti F, Romero CE, Gandhi CD. Isolated intraventricular hemorrhage associated with cerebral vasospasm and delayed cerebral ischemia following arteriovenous malformation rupture. Intervent Neurol. 2018;7(6):479-489.

19. Bambakidis NC, Cockroft K, Hu YC, et al. Procedural requirements and certification paradigms for stroke care delivery: perspective of neurointerventional professional societies. Stroke. 2017;48(10):2901-2904.

20. McIntyre MK, Halabi M, Li B, et al. Glycemic indices predict outcomes after aneurysmal subarachnoid hemorrhage: a retrospective single center comparative analysis. Sci Rep. 2021;11(1):158.

21. Lantigua H, Ortega-Gutierrez S, Schmidt JM, et al. Subarachnoid hemorrhage: who dies, and why? Crit Care. 2015; 19(1):309.

22. Perrein A, Petry L, Reis A, Baumann A, Mertes P, Audibert G. Cerebral vasospasm after traumatic brain injury: an update. Minerva Anestesiol. 2015;81(11):1219-1228.

23. Martin NA, Doberstein C, Zane C, Caron MJ, Thomas K, Becker DP. Posttraumatic cerebral arterial spasm: transcranial Doppler ultrasound, cerebral blood flow, and angiographic findings. J Neurosurg. 1992;77(4):575-583.

\section{Disclosures}

Dr. Kinon: honoraria from Globus Medical. 


\section{Author Contributions}

Conception and design: Al-Mufti, Dicpinigaitis. Acquisition of data: Dicpinigaitis. Analysis and interpretation of data: all authors. Drafting the article: Dicpinigaitis, Feldstein, Damodara, Cooper, Shapiro, Kamal, Kinon, Pisapia, Rosenberg, Gandhi. Critically revising the article: all authors. Reviewed submitted version of manuscript: all authors. Approved the final version of the manuscript on behalf of all authors: Al-Mufti. Statistical analysis: Dicpinigaitis. Administrative/technical/material support: Al-Mufti. Study supervision: Al-Mufti, Gandhi.

\section{Correspondence}

Fawaz Al-Mufti: Westchester Medical Center at New York Medical College, Valhalla, NY. fawaz.al-mufti@wmchealth.org. 